\title{
VIROLOGICAL SURVEILLANCE OF INFLUENZA IN FOUR RECENT POST-PANDEMIC SEASONS (2010/11 TO 2013/14) IN BULGARIA
}

\author{
Neli Korsun, Svetla Angelova, Ani Teodosieva \\ National Reference Laboratory "Influenza and ARD", National Centre of Infectious and Parasitic Diseases, Sofia, Bulgaria
}

\section{SUMMARY}

Aim: Influenza virological surveillance is an essential tool for studying the evolution of influenza viruses as well as for annual updating of the vaccine composition. The aim of the present study is to analyse the circulation of the influenza viruses in Bulgaria during the four recent postpandemic seasons.

Methods: A total of 3,681 respiratory samples from patients with influenza like illness (ILI) or acute respiratory illness (ARI) were tested for influenza viruses using Real Time RT-PCR.

Results: Influenza viruses were detected in 1,367 (37\%) samples. Of those viruses, $941(69 \%)$ were of type A and $426(31 \%)$ of type B. Among the subtyped A viruses, $543(60 \%)$ were $A(H 1 N 1) p d m 09$ and $369(40 \%) A(H 3 N 2)$. Co-circulation of all seasonal influenza types/subtypes was registered during each season, with the exception of $\mathrm{A}(\mathrm{H} 1 \mathrm{~N} 1) \mathrm{pdm} 09$ virus in the 2011/12 season. In this study, data gathered from the antigenic and genetic analyses of influenza viruses, their antiviral susceptibility, and the epidemiological and clinical characteristics of the infections are presented.

Conclusions: Yearly variations in the distribution and frequency of influenza types/subtypes and an annual shift of the predominant type/subtype were observed. In the seasons with predominant spread of $\mathrm{A}(\mathrm{H} 1 \mathrm{~N} 1)$ pdm09 virus - 2010/11 and 2013/14, a greater number of influenza-related pneumonia cases, ICU admissions and fatal cases was registered $(p<0.05)$. The results of the present study confirm the need for continuous and comprehensive influenza surveillance.

Key words: influenza, antigenic and genetic characterizations, pneumonia, neurologic complications

Address for correspondence: N. Korsun, Department of Virology, National Centre of Infectious and Parasitic Diseases, 44 Stoletov Blvd, 1233 Sofia, Bulgaria. E-mail: neli_korsun@abv.bg

http://dx.doi.org/10.21101/cejph.a4297

\section{INTRODUCTION}

Influenza is an acute, highly contagious viral respiratory infection that annually causes an enormous number of diseases, outpatient visits and hospitalizations, thus posing a significant burden on healthcare systems and the society as a whole. The annual attack rate in influenza is estimated at $5-10 \%$ in adults and $20-30 \%$ in children. Seasonal influenza epidemics account for 3 to 5 million cases of severe diseases, and between 250,000 and 500,000 deaths annually worldwide (1). Since 1977 , seasonal flu has been caused by influenza viruses $\mathrm{A}(\mathrm{H} 1 \mathrm{~N} 1), \mathrm{A}(\mathrm{H} 3 \mathrm{~N} 2)$ and type $\mathrm{B}$, which undergo constant antigenic and genetic changes. There are also temporal and geographical variations in the frequency of their distribution, virulence, clinical activity, and characteristics of the epidemic process. Periodically, every $10-40$ years, influenza type A viruses cause pandemics due to the emergence of fundamentally new subtype/variant influenza viruses against which there is no pre-existing immunity in the society.

The first pandemic in the 21st century was in 2009-2010 and was caused by a unique quadruple reassortant virus subtype $\mathrm{A}(\mathrm{H} 1 \mathrm{~N} 1)$, including a complex combination of swine, avian and human influenza virus genes. The pandemic $\mathrm{A}(\mathrm{H} 1 \mathrm{~N} 1) \mathrm{pdm} 09$ virus continued to circulate in the following years and fully replaced seasonal influenza viruses of the same subtype. The global influenza virological surveillance is conducted by the World Health Organization (WHO) so as to monitor the evolution of influenza viruses and to alert for the emergence of influenza viruses with pandemic potential. The timely detection of circulating influenza viruses, the determination of their antigenic, genetic and biological properties, including their susceptibility to available antiviral drugs, the rapid identification of antigenic drift variants and novel A subtypes with pandemic potential as well as the analysis of clinical and epidemiological aspects of infections are important tasks of the national surveillance systems (2-4).

This report presents results of the influenza virus surveillance performed during the four recent post-pandemic seasons (2010/11 to 2013/14) in Bulgaria.

\section{MATERIALS AND METHODS}

\section{Study Population}

A total of 3,681 patients, who were ambulatory treated or hospitalized either for ILI or for ARI in different regions of the country, were enrolled. The ECDC case definitions of ILI and 
ARI were used to identify eligible patients (5). Each patient's diagnosis based on standard clinical criteria was determined by attending physician. Each clinical specimen was accompanied by a case report form filled in with epidemiological and clinical data: demographic characteristics, history of illness, symptoms, initial diagnosis, underlying conditions, and antiviral treatment.

\section{Specimen Collection}

Respiratory specimens from 10-20 ARI/ILI patients attending each primary care sentinel site were collected at the start of the relevant season. All hospitalized severe ILI cases in 10 hospitals (infectious disease hospitals/wards, paediatric hospitals/wards, pulmonary care units, intensive care units) in the country were sampled throughout the season. Combined nasal and pharyngeal specimens from the enrolled patients were collected with the help of commercial polyester swabs (DeltaLab, Spain). Swabs were stored at $4^{\circ} \mathrm{C}$ for up to 72 hours before shipment to the Laboratory. Specimens were processed immediately or stored at $-80^{\circ} \mathrm{C}$ before testing.

\section{Extraction of Nucleic Acids and Real Time RT-PCR}

In the first two seasons reported herein, viral nucleic acids were extracted automatically from respiratory specimens using a commercial iPrep Pure Link Virus Kit (Invitrogen, USA), while in the third and in the fourth seasons, ExiPrep Dx Viral DNA/RNA kit (Bioneer, Korea) was used, both applied according to the manufacturer's instructions. Laboratory testing was conducted at the National Laboratory "Influenza and ARD", which is recognized by the WHO as the National Influenza Centre. The Laboratory undergoes annual quality control assessment conducted by WHO EQAP and INSTAND-Germany. The detection and the typing/ subtyping of influenza viruses were carried out by a Real Time RT-PCR method with the use of a kit SuperScript III Platinum ${ }^{\circledR}$ One-Step Quantitative RT-PCR System (Invitrogen, USA). All samples were first tested for influenza A and B viruses, and those positive for influenza A were subsequently tested for $\mathrm{A}(\mathrm{H} 1 \mathrm{~N} 1)$ pdm09 and A(H3N2). Primers, probes and positive controls for influenza A/B viruses were provided by the CDC-Atlanta. The amplification was performed with a Chromo 4 thermal cycler (Bio-Rad) in accordance with the protocol of CDC-Atlanta (reverse transcription at $50^{\circ} \mathrm{C}$ for 30 minutes, Taq inhibitor activation at $95^{\circ} \mathrm{C}$ for 2 minutes, followed by the 45 cycles of denaturation at $95^{\circ} \mathrm{C}$ for 15 seconds and annealing at $55^{\circ} \mathrm{C}$ for 30 seconds) (6). A Ct value $<38$ was regarded as positive.

\section{Antigenic and Genetic Characterization}

The isolation of influenza viruses was carried out on MDCK and MDCK-SIAT-1 cell cultures. The antigenic characterization of isolates was performed by the haemagglutination inhibition (HI) assay, in accordance with the WHO manual, using vaccine viruses/ antigens and their corresponding antisera received from the World Collaborating Centre for Influenza (WHO-CC), London and CDC, Atlanta (7). First, reference antisera were treated by a receptor destroying enzyme (RDE) to inactivate nonspecific inhibitors of haemagglutination. Then in 96-well U-bottom microtitre plates, a standardized quantity of haemagglutinin (HA) antigen (8 hae- magglutination units $/ 50 \mu \mathrm{l}$ ) was mixed with an equal volume of serially two-fold diluted antiserum. After incubation for $30 \mathrm{~min}$, $50 \mu 11 \%$ guinea-pig red blood cells were added to all wells. The $\mathrm{HI}$ titre was determined as the reciprocal of the highest dilution of antiserum that completely inhibits haemagglutination. A viral isolate was identified as antigenically related to the vaccine virus if it showed no more than 4-fold reduced HI titre with antiserum raised against the vaccine virus, as compared to the titre for the vaccine virus. More detailed HI assay with a panel of reference strains and antisera, as well as sequencing of hemagglutinin (HA) and neuraminidase (NA) genes of representative Bulgarian influenza isolates, were performed at the WHO-CC, London.

\section{Antiviral Susceptibility Monitoring}

Screening of $\mathrm{A}(\mathrm{H} 1 \mathrm{~N} 1) \mathrm{pdm} 09$ viruses for the detection of $\mathrm{H} 275 \mathrm{Y}$ oseltamivir resistance point mutation was carried out using a Real Time RT-PCR discrimination assay. Primer/probe sequences and protocol were kindly provided by the Health Protection England (HPE), London. Phenotypic analysis (MUNANA test) of influenza virus susceptibility to neuraminidase inhibitors (oseltamivir and zanamivir) was performed at the WHO-CC, London.

\section{Statistics}

Age and gender of the patients, clinical features of the illness and the incidence of each virus were compared using Chi square or Fisher's exact tests for categorical variables. The two-tailed Student's t-test was used for comparison of continuous variables in two different groups. P value of $<0.05$ was considered statistically significant.

\section{RESULTS}

The ARI surveillance system was implemented to monitor influenza in Bulgaria. The National Influenza Surveillance Programme is coordinated by the National Reference Laboratory "Influenza and ARD" in collaboration with the Department of Epidemiology of the National Centre of Infectious and Parasitic Diseases. Since 2007 a web-based information system has been used for sentinel surveillance of influenza and ARI in Bulgaria. The epidemiological and virological surveillance are integrated into the system. The surveillance of ARI is carried out by a national sentinel network of general practitioners and paediatricians working at 208 healthcare facilities situated in all 28 major cities - regional centres covering $5.3 \%$ of the general population in the country. Primary care physicians report weekly the numbers of clinical cases of ARI within five age categories ( $0-4$ years, 5-14 years, 15-29 years, 30-64 years, and 65+ years), collect respiratory specimens and send them to the National Reference Laboratory for testing in order to obtain information on the types/ subtypes and strains of influenza viruses circulating in the country.

This study covers four winter epidemic seasons consisting of 33 calendar weeks each (starting from week 40 of the previous year to week 20 of the next year). During these seasons, the first increases in ARI consultation rates were reported in December. ARI consultation rates reached their highest levels in late January 
to early February and returned to normal out-of-winter season levels by mid-April. The highest numbers of outpatient visits due to ARI were seen in the age groups of $0-4$ and 5-14 years. The sentinel based estimate of ARI incidence was highest in the 2010/11 season and lowest in the 2012/13 season (8).

The study population consisted of 3,681 patients: 813 people in the first, 588 in the second, 1,123 in the third and 1,157 people in the fourth season. A total of 932 (25\%) of these were patients attending outpatient healthcare centres, 2,561 (70\%) were hospitalized patients, and $188(5 \%)$ patients admitted to intensive care units (ICU). The patients' age ranged from 15 days to 92 years. Among the study subjects, $862(23 \%)$ were aged $0-4$ years; 740 (20\%) 5-14 years; 495 (13\%) 15-29 years; 1,147 (31\%) 30-64 years; and $376(10 \%) \geq 65$ years; while $61(1.7 \%)$ were at unknown age. Most patients $(72 \%)$ demonstrated symptoms of ILI or ARI without serious complications. However, some complications in the lower respiratory tract (bronchiolitis, pneumonia) and in the central nervous system (CNS) (febrile seizures, oedema cerebri, aseptic meningitis, encephalopathy, meningoencephalitis) were analysed.

\section{Influenza Virus Detection}

Influenza infection was laboratory confirmed in 1,367 (37\%) patients' samples: 399 (49\%), 247 (42\%), 331 (29\%), and 390 (34\%) in the 2010/11, 2011/12, 2012/13 and 2013/14 seasons, respectively. A total of $941(69 \%)$ specimens were found positive for influenza type A virus, 426 (31\%) for type B, 29 (2\%) were type A unsubtyped. Among the subtyped A viruses, 543 (60\%) were A(H1N1)pdm09 and 369 (40\%) A(H3N2).

During the first post-pandemic season, namely season 2010/11, influenza A(H1N1)pdm09 was the predominant virus detected in $222(27 \%)$ of all patients' samples, followed by type B identified in $169(21 \%)$ samples. In the next 2011/12 season, influenza A(H3N2) was predominantly detected in 243 (41\%) patients' samples, A(H1N1)pdm09 virus was not detected. During the 2012/2013 season, all 3 seasonal influenza viruses co-circulated but influenza $\mathrm{B}$ was the predominant circulating strain. In that season, 60 (5\%) influenza detections were A(H1N1)pdm09, $21(2 \%) \mathrm{A}(\mathrm{H} 3 \mathrm{~N} 2)$, and $250(22 \%)$ type B. The last season was dominated by A(H1N1)pdm09 (23\%), followed by A(H3N2) (9\%), while type B viruses were detected very rarely (Fig. 1).

The distribution of detected influenza viruses in outpatients and inpatients is presented in Fig. 2. The incidence of A(H1N1)pdm09 in the 2010/11 season and type B in the 2010/11 and 2012/13 seasons was higher in inpatients than that in outpatients $(30.1 \%$ vs. $37 \%$; and $27.7 \%$ vs. $11.5 \%, 10.7 \%$ vs. $10.5 \%$, respectively). The detection rate of A(H1N1)pdm09 in the 2013/14 season was higher in outpatients $(40.7 \%$ vs. $19.7 \%)(\mathrm{p}<0.05)$.

\section{Age Distribution}

The mean age of patients infected by the different influenza viruses, i.e. $\mathrm{A}(\mathrm{H} 1 \mathrm{~N} 1) \mathrm{pdm} 09, \mathrm{~A}(\mathrm{H} 3 \mathrm{~N} 2)$ and type $\mathrm{B}$, was $32.12 \pm 23.49$ years, $22.04 \pm 24.53$ years and $22.24 \pm 2.03$ years, respectively. In the 2010/2011 season, the groups aged 30-64 and 5-14 years had the highest incidence rates for $\mathrm{A}(\mathrm{H} 1 \mathrm{~N} 1)$ pdm09 (34\%) and type B viruses (41\%), respectively. In the 2011/2012 season, the predominant A(H3N2) virus was most prevalent among 5-14 and 0-4 years old children $-51 \%$ and $48 \%$, respectively. Influenza B viruses, which dominated the next 2012/13 season, were more commonly found among the 5-14 year-olds ( $34 \%)$. The youngest children ( $0-4$ years) and middleaged adults (30-64 years) were most frequently affected by the predominant $\mathrm{A}(\mathrm{H} 1 \mathrm{~N} 1)$ pdm09 virus in the last epidemic season
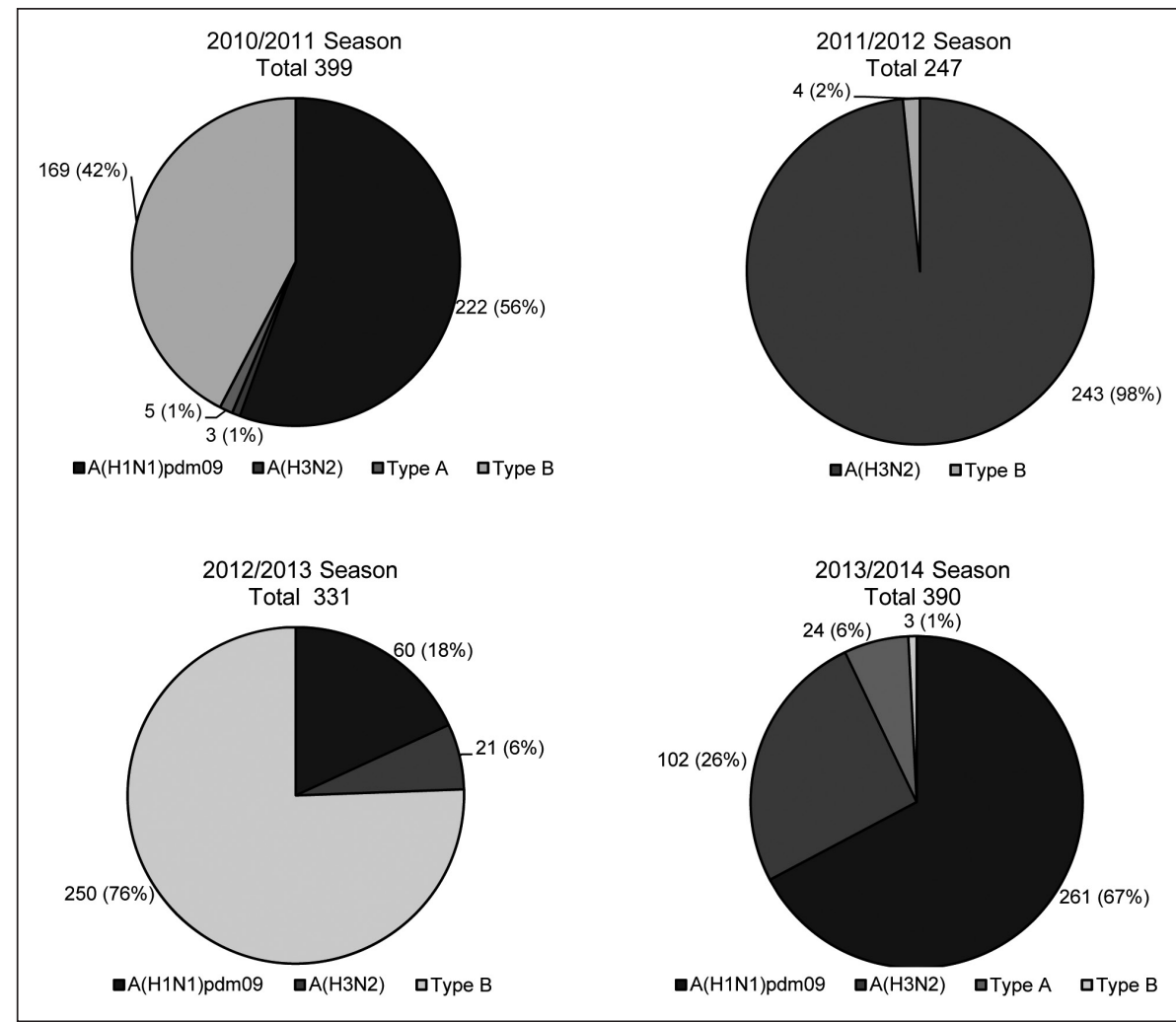

Fig. 1. Number (\%) of detected influenza viruses. 


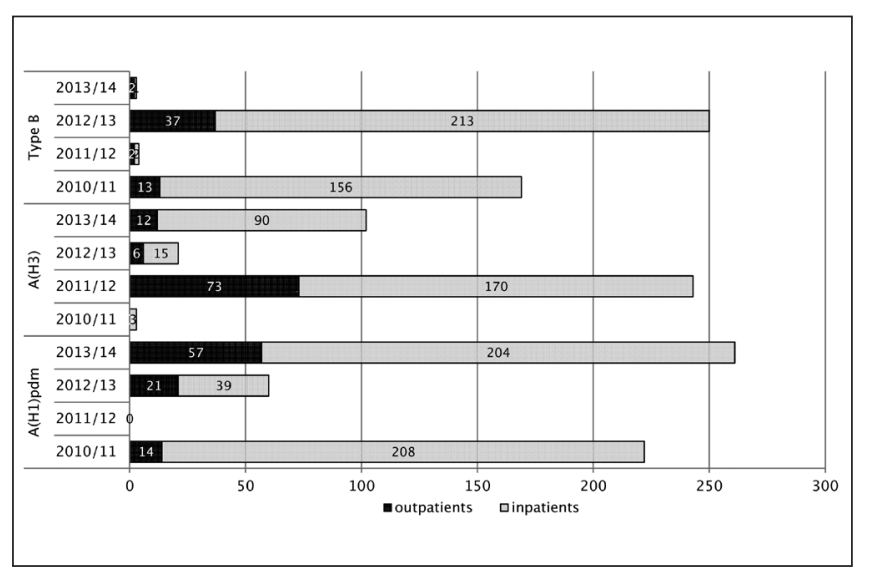

Fig. 2. Number of detected influenza viruses in outpatients and inpatients.

(Table 1). Generally, during all seasons A(H1N1)pdm09 virus was detected most often in middle-aged adults (30-64 years), among both outpatients and hospitalized patients; A(H3N2) virus in subjects in the extreme age groups $(\geq 65$ years old and children), and type B virus in 5-14 years old children. Among patients admitted to ICU, those with the highest percentage of $\mathrm{A}(\mathrm{H} 1 \mathrm{N1}) \mathrm{pdm} 09$ virus infection were those aged 30-64 and 15-29. Over the study period, A(H3N2) virus infection was not proven in ICU patients (Table 2).

\section{Monthly Distribution}

During the different reported flu seasons, the detection of influenza viruses started in weeks 49/2010, 2/2012, 3/2013 and $50 / 2013$ and lasted until weeks 17/2011, 18/2012, 20/2013 and $15 / 2013$. The influenza virus activity peaked in weeks $4 / 2011$, 5/2012, 7/2013 and 4/2014 (Fig. 3).

\section{Antigenic Strain Characterization}

During the four periods covered by the study, a total of 103 influenza viruses were isolated on cell cultures and submitted to the WHO-CC, London. All 64 A(H1N1)pdm09 strains were antigenically related to the vaccine strain A/California/07/2009. Among the $26 \mathrm{~A}(\mathrm{H} 3 \mathrm{~N} 2)$ isolates tested by HI assay, 25 (96\%) showed 8 -fold or greater reductions with the antisera raised against the actual egg-propagated vaccine strains. All 13 influenza B strains of the 2012/13 season belonged to the B/ Yamagata/16/88-lineage. In HI assay two B viruses had homology to the 2012/2013 vaccine component, B/Wisconsin/1/2010, and $11 \mathrm{~B}$ viruses showed relation to the vaccine strain $\mathrm{B} /$ Massachusetts/2/2012, which had been recommended for the following 2013/2014 season.

\section{Genetic Strain Characterization}

During the first surveillance period 2010/11, two of the A(H1N1)pdm09 viruses sequenced belonged to genetic group 6 . Four of the sequenced A(H1N1)pdm09 viruses of the 2012/2013 season belonged to genetic subgroup $6 \mathrm{C}$, and all 19 sequenced pandemic viruses of the 2013/14 season to genetic subgroup 6B (Fig. 4). A(H3N2) isolates sequenced in the 2011/12 season, fell

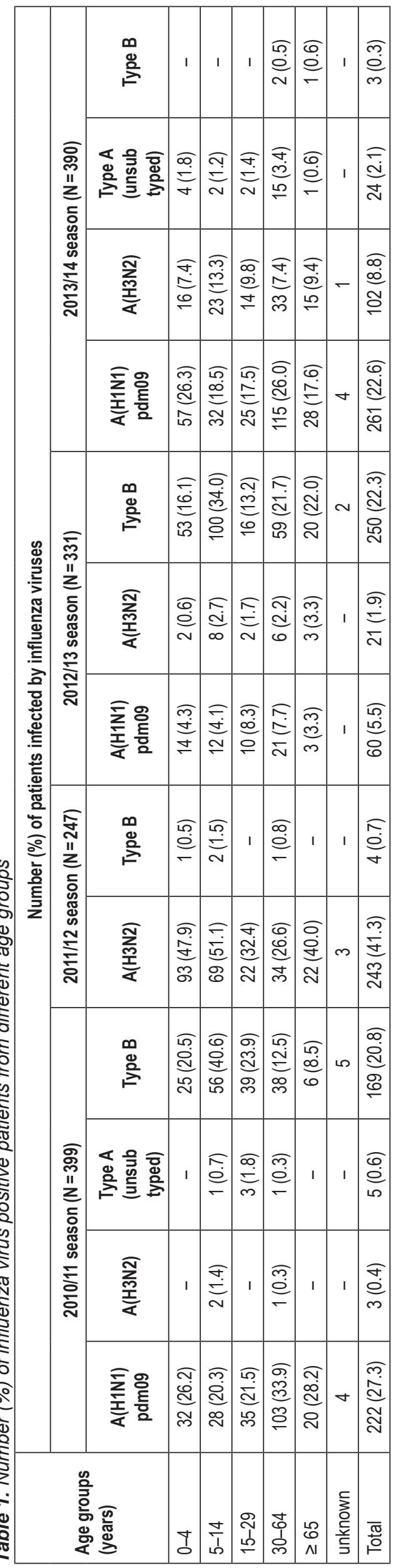


Table 2. Comparisons of numbers (\%) of influenza $A(H 1 N 1) 2009, A(H 3 N 2)$ and type $B$ cases among different age groups

\begin{tabular}{|c|c|c|c|c|}
\hline $\begin{array}{l}\text { Age groups } \\
\text { (years) }\end{array}$ & No. of patients tested & $\begin{array}{c}\mathrm{A}(\mathrm{H} 1 \mathrm{N1} \text { 1)pdm09 } \\
\mathrm{n}(\%)\end{array}$ & $\begin{array}{c}\mathrm{A}(\mathrm{H} 3 \mathrm{~N} 2) \\
\mathrm{n}(\%)\end{array}$ & $\begin{array}{l}\text { Type B } \\
\text { n (\%) }\end{array}$ \\
\hline \multicolumn{5}{|l|}{ Outpatients } \\
\hline $0-4$ & 235 & $21(8.9)$ & $29(12.3)$ & $8(3.4)$ \\
\hline $5-14$ & 292 & $24(8.2)$ & $33(11.3)$ & $29(9.9)$ \\
\hline $15-29$ & 117 & $13(11.1)$ & $7(6)$ & $4(3.4)$ \\
\hline $30-64$ & 241 & $33(13.7)$ & $15(6.2)$ & $13(5.4)$ \\
\hline$\geq 65$ & 47 & $3(6.4)$ & $6(12.8)$ & $1(2.1)$ \\
\hline \multicolumn{5}{|c|}{ Hospitalized patients } \\
\hline $0-4$ & 611 & $81(13.3)$ & $82(13.4)$ & $69(11.3)$ \\
\hline $5-14$ & 437 & $47(10.8)$ & $69(15.8)$ & $129(29.5)$ \\
\hline $15-29$ & 360 & $51(14.2)$ & $31(8.6)$ & $49(13.6)$ \\
\hline $30-64$ & 795 & $161(20.3)$ & $59(7.4)$ & $76(9.6)$ \\
\hline$\geq 65$ & 297 & $37(12.5)$ & $34(11.4)$ & $22(7.4)$ \\
\hline \multicolumn{5}{|l|}{ ICU patients } \\
\hline $0-4$ & 16 & $1(6.3)$ & - & $1(6.3)$ \\
\hline $5-14$ & 11 & - & - & - \\
\hline $15-29$ & 18 & $6(33.3)$ & - & $1(5.6)$ \\
\hline $30-64$ & 111 & $36(34)$ & - & $10(9)$ \\
\hline$\geq 65$ & 32 & $8(25)$ & - & $3(9.4)$ \\
\hline
\end{tabular}

into genetic groups 6 (10 strains) and subgroup 3A (2 strains). Both sequenced Bulgarian A(H3N2) isolates of the 2012/13 season belonged to subgroup $3 \mathrm{C}$. $\mathrm{A}(\mathrm{H} 3 \mathrm{~N} 2)$ strains isolated in the 2013/14 season fell into genetic groups 3C.2 (one strain) and 3 C. 3 ( 7 strains). Among the sequenced influenza B viruses in the 2012/13 season, all 6 strains fell into genetic clade 2.

\section{Antiviral Susceptibility}

All 60 A(H1N1)pdm09 viruses detected in the 2012/13 season and $261 \mathrm{~A}(\mathrm{H} 1 \mathrm{~N} 1)$ pdm09 viruses identified in the 2013/14 season, were analysed by Real-Time RT-PCR with respect to the H275Y amino acid substitution, which is linked to oseltamivir resistance. Only one of the strains carried the substitution. All 103 influenza virus isolates analysed phenotypically in the WHO-CC, London, were sensitive to the neuraminidase inhibitors oseltamivir and zanamivir.

\section{Clinical Characteristics}

The most serious complications of influenza illness are associated with the lower respiratory tract and CNS. The contributing

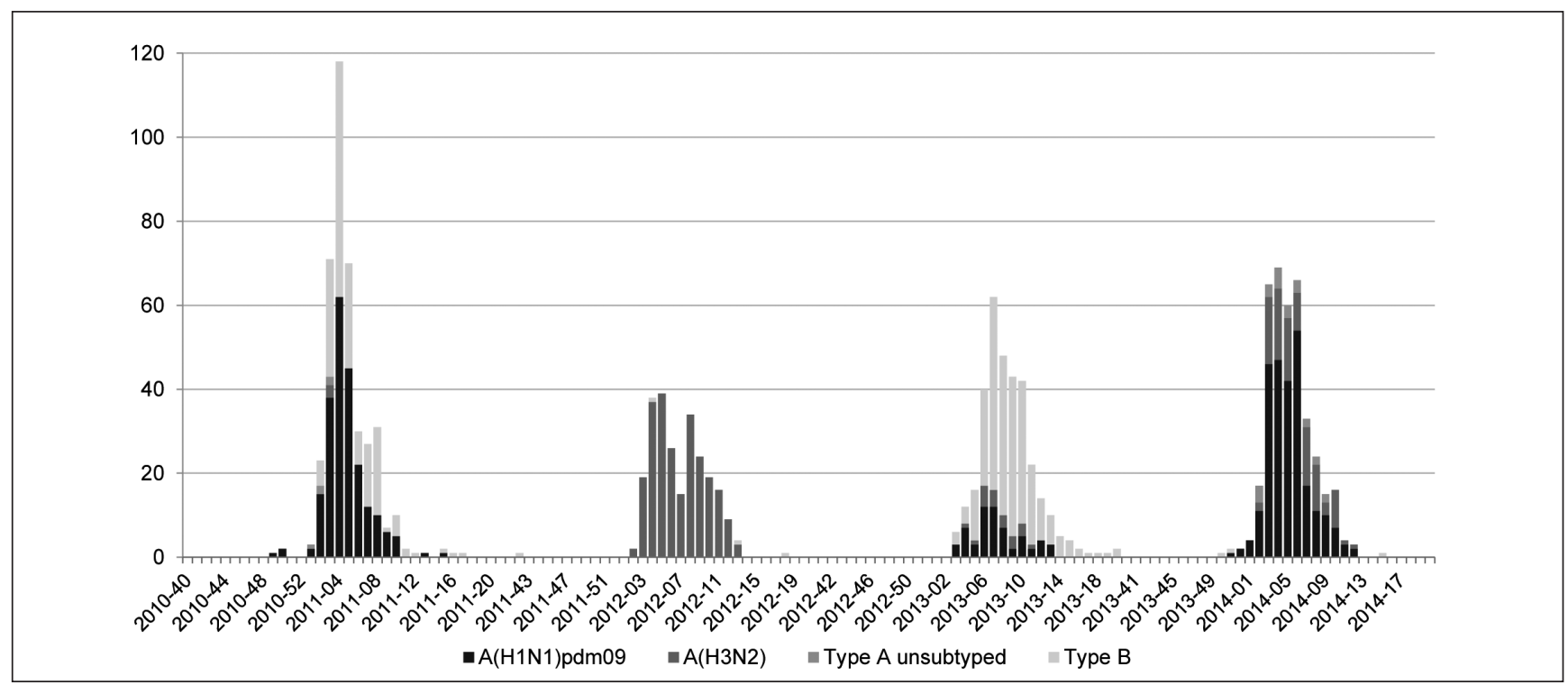

Fig. 3. Temporal distribution of influenza virus detections. 
Table 3. Distribution of detected influenza viruses according to clinical diagnosis of patients

\begin{tabular}{|c|c|c|c|c|c|}
\hline \multirow[b]{2}{*}{ Seasons } & \multirow{2}{*}{$\begin{array}{c}\text { Detected influenza } \\
\text { viruses }\end{array}$} & \multicolumn{4}{|c|}{ Clinical characteristics } \\
\hline & & $\begin{array}{l}\text { Bronchiolitis } \\
\quad(n=36)\end{array}$ & $\begin{array}{l}\text { Pneumonia } \\
(n=623)\end{array}$ & $\begin{array}{l}\text { CNS symptoms } \\
\quad(n=371)\end{array}$ & $\begin{array}{l}\text { Fatal cases } \\
\qquad(n=29)\end{array}$ \\
\hline \multicolumn{2}{|c|}{ Mean age $\pm S D^{*}(y)$ of infected patients } & $1.92 \pm 2.02$ & $39.09 \pm 23.39$ & $22.52 \pm 20.99$ & $46.71 \pm 22.28$ \\
\hline \multicolumn{2}{|c|}{ Male/female ratio among infected patients } & $8 / 5$ & $121 / 100$ & $52 / 54$ & $10 / 7$ \\
\hline \multirow[t]{4}{*}{ 2010/11 } & A(H1N1)pdm09 & 2 & $79(35.7 \%)$ & $9(18.4 \%)$ & 10 \\
\hline & $\mathrm{A}(\mathrm{H} 3 \mathrm{~N} 2)$ & - & $1(0.5 \%)$ & - & - \\
\hline & Type A unsubtyped & - & $1(0.5 \%)$ & $1(2 \%)$ & 1 \\
\hline & Type B & - & $28(12.7 \%)$ & $20(40.8 \%)$ & 2 \\
\hline \multirow[t]{2}{*}{ 2011/12 } & $\mathrm{A}(\mathrm{H} 3 \mathrm{~N} 2)$ & 3 & $13(21.3 \%)$ & $10(14.5 \%)$ & - \\
\hline & Type B & 1 & $1(1.6 \%)$ & $1(1.4 \%)$ & - \\
\hline \multirow[t]{3}{*}{ 2012/13 } & $\mathrm{A}(\mathrm{H} 1 \mathrm{~N} 1) \mathrm{pdm09}$ & - & $10(7.7 \%)$ & $6(5 \%)$ & 1 \\
\hline & $\mathrm{A}(\mathrm{H} 3 \mathrm{~N} 2)$ & 1 & $1(0.8 \%)$ & $5(4.2 \%)$ & - \\
\hline & Type B & 3 & $19(14.6 \%)$ & $22(18.3 \%)$ & 1 \\
\hline \multirow[t]{3}{*}{ 2013/14 } & A(H1N1)pdm09 & 3 & $56(26.5 \%)$ & $23(17.3 \%)$ & 1 \\
\hline & $\mathrm{A}(\mathrm{H} 3 \mathrm{~N} 2)$ & - & $10(4.7 \%)$ & $10(7.5 \%)$ & - \\
\hline & Type A unsubtyped & - & $5(2.4 \%)$ & - & 1 \\
\hline Total & $13(36.1 \%)$ & $224(36 \%)$ & $107(28.8 \%)$ & $17(58.6 \%)$ & \\
\hline
\end{tabular}

${ }^{*} \mathrm{SD}$ - standard deviation

role of influenza viruses in cases of bronchiolitis, pneumonia, CNS symptoms and fatalities is analysed herein. During seasons 2010/11, 2011/12, 2012/13, 2013/14, the number of investigated patients with bronchiolitis was $4,11,9,12$, respectively; with pneumonia 221, 61, 130, 211; with CNS symptoms 49, 69, 120, 133; and those with a fatal outcome $17,1,3,8$, respectively. Among the patients with pneumonia, 123 (20\%) required intensive care and $12(2 \%)$ passed away; among the patients with CNS complications, 15 (14\%) were admitted to ICU and 1 passed away. The individuals with different clinical diagnosis differed significantly in terms of age (Table 3). The mean age of patients with bronchiolitis was the lowest one, and the mean age of patients with pneumonia the highest. Among influenza-positive patients with pneumonia, the highest percentage (47\%) represented the 30-64 year-olds, and the lowest (8\%) the 5-14 year-olds. Among the patients with CNS symptoms, the influenza virus positivity was highest (34\%) in the group aged 5-14 and lowest (5\%) in patients aged $\geq 65(p<0.05)$. Male predominance was found in patients with pulmonary complications and fatal cases. The proportion of influenza virus detections among patients with bronchiolitis was $13 / 36(36 \%)$, pneumonia 224/623 (36\%), and neurological complications $107 / 371$ (29\%). The most frequent causative agents of pneumonia were $\mathrm{A}(\mathrm{H} 1 \mathrm{~N} 1)$ pdm09 (2010/11 and 2013/14 seasons), A(H3N2) (2011/12 season) and type B (2012/13 season) $(p<0.05)$. Influenza type B viruses were proved unusually often in patients with neurological symptoms during the 2010/11 season. The greatest number of ICU patients (87) and fatal cases (17) was registered in the first post-pandemic season. Among the patients admitted to ICU, 47\% were positive for influenza. In the last 2013/14 season, there was a great number of ICU cases (56) and fatalities (8), as well. Totally, 15 (59\%) influenza viruses were detected in deceased patients - 11 A(H1N1)pdm09, 3 type B and 1 type A unsubtyped.

\section{DISCUSSION}

This study provides an analysis of the circulation of influenza viruses, their antigenic and genetic characteristics, the epidemiological patterns and clinical features of infections during the four post-pandemic seasons in Bulgaria. Co-circulation of all seasonal influenza types/subtypes was registered during each season with the exception of A(H1N1)pdm09 virus in the 2011/12 season. The detection rates of influenza type A were higher as compared to those of type B viruses, except for the 2012/13 season. Influenza $\mathrm{A}(\mathrm{H} 1 \mathrm{~N} 1) \mathrm{pdm} 09, \mathrm{~A}(\mathrm{H} 3 \mathrm{~N} 2)$ and type B dominated in different periods with a small overlap. Yearly variations in the distribution and frequency of influenza types/subtypes and an annual shift of the dominant influenza type/subtype were observed. Seasons of low circulation of a particular type/subtype were followed by seasons of its increased circulation and vice versa. Constant changes in the antigenic properties of the circulating influenza viruses and in the immune status of the population were suspected as the reason for that observation. The influenza vaccine coverage in Bulgaria is very low (2-3\%), and the vaccine status probably does not affect the circulation of different types/subtypes of influenza viruses. Our data concerning trends of influenza virus circulation are similar to those of other European countries (8-12).

During the four-year study period, the antigenic analysis of $\mathrm{A}(\mathrm{H} 1 \mathrm{~N} 1) \mathrm{pdm} 09$ isolates revealed a close relationship with the vaccine component $\mathrm{A} / \mathrm{California} / 7 / 2009$. The high incidence rate of A(H1N1)pdm09 virus infections in the 2010/11 and 2013/14 seasons did not result from virus evolutionary changes, and the presence of large contingents of people who had not suffered such an infection during the previous season. The antigenic and sequence profiles of most $\mathrm{A}(\mathrm{H} 3 \mathrm{~N} 2)$ viruses isolated in cell cultures demonstrate an antigenic difference with the actual vaccine strains and annual genetic changes. This can be explained by the 


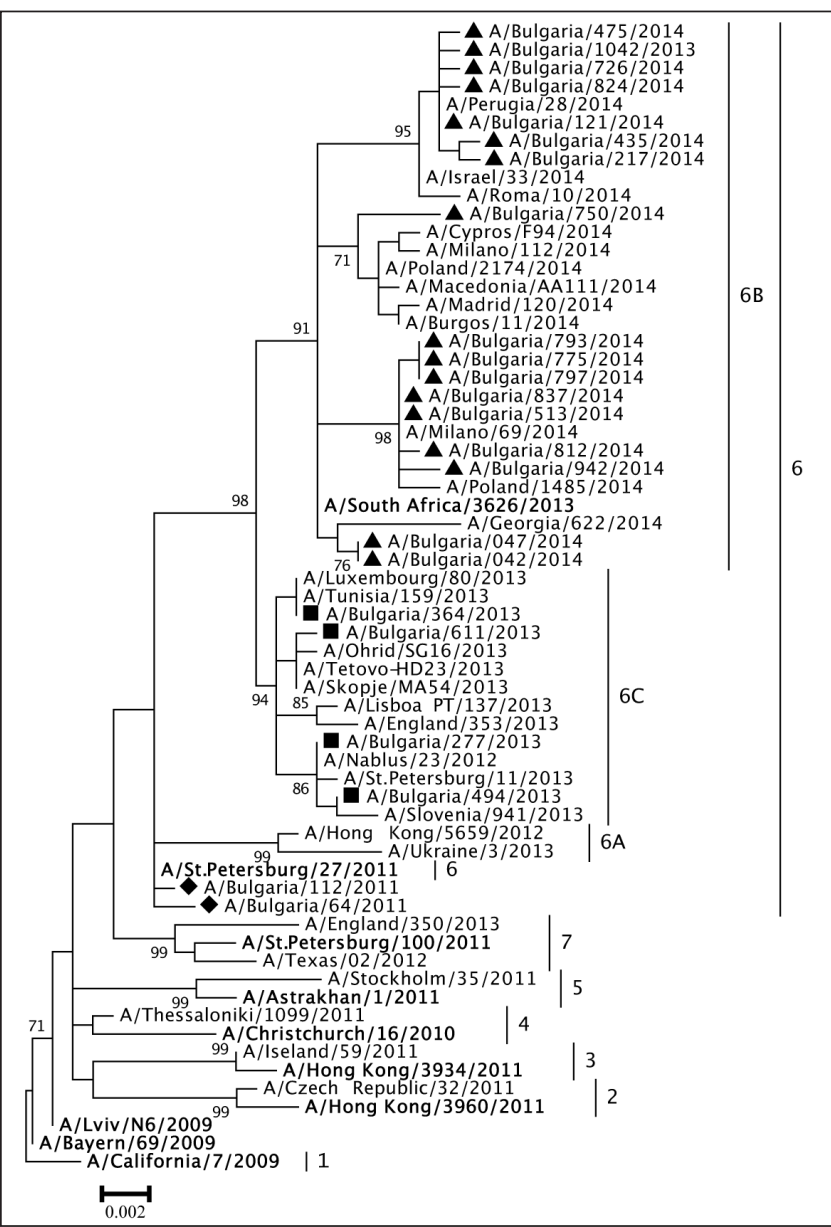

Fig. 4. Phylogenetic analysis of the HA genes of circulating influenza $A(H 1 N 1) p d m 09$ viruses. The phylogenetic tree was constructed using the Maximum Likelihood algorithm with 1000 bootstrapping replicates, HKY model and MEGA6 software. Only bootstrap values $\geq 70 \%$ are shown. The selected worldwide sequences were retrieved from GISAID website (25). The Bulgarian sequences from the 2010/2011, 2012/2013 and 2013/2014 seasons are indicated by solid roundels, squares and triangles, respectively; the reference strains representing known genetic groups are displayed in bold. Genetic groups/ subgroups are shown in lines.

fact that $\mathrm{A}(\mathrm{H} 3 \mathrm{~N} 2)$ viruses are the most quickly evolving influenza ones, and that in the process of reproduction in hens' eggs, the vaccine virus strains produce adaptive mutations in the HA gene. Therefore, egg-derived vaccine strains and mammalian (MDCK) cell-derived isolates show very different levels of reactivity to post-infection ferret antisera as determined by the HI assay (13, 14). The incomplete match between $\mathrm{A}(\mathrm{H} 3 \mathrm{~N} 2)$ vaccine strains and the circulating influenza viruses is the basis for the observed lower vaccine effectiveness against $\mathrm{A}(\mathrm{H} 3 \mathrm{~N} 2)$ viruses (15). Bulgarian influenza strains of different types/subtypes belong to the most common genetic groups in Europe (8).

All but one Bulgarian influenza viruses tested were sensitive to neuraminidase inhibitors. Globally, oseltamivir resistance of $\mathrm{A}(\mathrm{H} 1 \mathrm{~N} 1)$ pdm09 viruses due to the H275Y mutation in neuraminidase gene accounts for $1-2 \%$ of the strains tested. Resistance in $\mathrm{A}(\mathrm{H} 3 \mathrm{~N} 2)$ and $\mathrm{B}$ viruses is extremely rare (9-12).

The age distribution of patients infected by different influenza viruses in this study confirms a fact observed earlier, namely that pandemic $\mathrm{A}(\mathrm{H} 1 \mathrm{~N} 1) \mathrm{pdm} 09$ virus characteristically infects children and middle-aged adults in comparison to influenza $A(H 3 N 2)$, where extreme age individuals are the more susceptible population groups (16-18). Type B viruses are more frequently detected in the 5-14 year-olds. Analysis of the age-specific distribution of influenza infection may be useful to public health professionals in determining priority groups for preventive measures.

The temporal distribution of influenza virus detections reveals slight differences at the beginning, at the end and over the duration of influenza seasons. The peaks in influenza incidence in January and February observed in this study coincide with the lower temperatures recorded during these months. The latter finding would contribute to determining the optimal periods for influenza vaccine implementation among priority population groups.

Influenza-related pneumonia and CNS manifestations are among the most serious clinical conditions leading to hospitalization and ICU admission. The association of influenza with acute pneumonia has been documented in many studies (19). According to several reports, up to $10 \%$ of patients with $\mathrm{A}(\mathrm{H} 1 \mathrm{~N} 1) \mathrm{pdm} 09$ infection demonstrate neurologic symptoms $(20,21)$. In the present study, the detection rate of influenza viruses among patients with bronchiolitis, pneumonia, CNS complications and fatal illness amounts to $36.1 \%, 36 \%, 28.8 \%$ and $58.6 \%$, respectively. Generally, the dominant in its distribution type/subtype emerges as the most common cause of bronchiolitis, pneumonia and neuroinfections in the respective season: $\mathrm{A}(\mathrm{H} 1 \mathrm{N1})$ pdm09 virus in the 2010/11 and 2013/14 seasons, A(H3N2) virus in the 2011/12 season, and type B viruses in the 2012/13 season. Influenza B viruses make an exception as in the 2010/11 season they were the more frequent cause of CNS complications as compared to the predominant A(H1N1) pdm09 virus. In the latter season, influenza B viruses caused severe infections associated with pneumonia, CNS complications and death. Unexpected severity of cases of influenza B infection in patients that required hospitalization was also observed in other countries during the first post-pandemic season (22). On the whole, in the seasons with predominant spread of A(H1N1)pdm09 virus 2010/11 and 2013/14, a greater number of influenza-related pneumonia cases, ICU admissions and fatal cases was registered $(p<0.05)$. Increased severity of influenza A(H1N1)pdm09 infection during the first post-pandemic season was also observed in the UK, Germany and other countries $(23,24)$.

This article is the first one in Bulgaria to present data of virological surveillance of influenza carried out by Real Time RT-PCR during the four post-pandemic seasons. The results obtained confirm the need for a continuous monitoring of seasonal influenza types/subtypes, which can help to determine trends in their circulation, seasonal patterns and the disease burden of infections, susceptible population groups, etc. The study of the genetic and antigenic changes of influenza viruses, as well as their susceptibility to antiviral drugs, is an extremely important task, especially in terms of control of epidemics and preparedness for a pandemic.

\section{Acknowledgements}

The authors would like to express their gratitude to Dr John McCauley and the staff at the WHO-CC, London, for their significant contribution to this study.

The study was carried out with the financial support of the Ministry of Health of Bulgaria. 


\section{Adherence to Ethical Recommendations}

The study was performed in accordance with the principles of the 1975 Helsinki Declaration and approved by the local Ethics Committee at the NCIPD. Patients provided a written informed consent prior to specimen collection and testing.

\section{Conflict of Interests}

None declared

\section{REFERENCES}

1. World Health Organization. Media centre. Influenza (seasonal) [Internet]. Geneva: WHO; 2014 [cited 2016 Mar 14]. Available from: http://www. who.int/mediacentre/factsheets/fs211/en/.

2. Badar N, Bashir Aamir U, Mehmood MR, Nisar N, Alam MM, et al. Influenza virus surveillance in Pakistan during 2008-2011. PLoS One. 2013 Nov 8;8(11):e79959. doi: 10.1371/journal.pone.0079959.

3. El Moussi A, Pozo F, Ben Hadj Kacem MA, Ledesma J, Cuevas MT, et al. Virological Surveillance of Influenza Viruses during the 2008-09, 2009-10 and 2010-11 Seasons in Tunisia. PLoS One. 2013 Sep 19;8(9):e74064. doi: 10.1371/journal.pone.0074064.

4. Chuang JH, Huang AS, Huang WT, Liu MT, Chou JH, Chang FY, et al. Nationwide surveillance of influenza during the pandemic (200910) and post-pandemic (2010-11) periods in Taiwan. PLoS One. 2012;7(4):e36120. doi: 10.1371/journal.pone.0036120.

5. European Centre of Disease Prevention and Control (ECDC). Influenza case definitions [Internet]. ECDC; 2016 [cited 2016 Mar 14]. Available from: http://www.ecdc.europa.eu/en/activities/surveillance/eisn/surveillance/pages/influenza_case_definitions.aspx.

6. Shu B, Wu KH, Emery S, Villanueva J, Johnson R, Guthrie E, et al. Design and performance of the CDC real-time reverse transcriptase PCR swine flu panel for detection of 2009 A (H1N1) pandemic influenza virus. J Clin Microbiol. 2011 Jul;49(7):2614-9.

7. WHO Global Influenza Surveillance Network. Manual for the laboratory diagnosis and virological surveillance of influenza [Internet]. Geneva: WHO; 2011 [cited 2016 Mar 14]. Available from: http://whqlibdoc.who. int/publications/2011/9789241548090 eng.pdf.

8. European Centre for Disease Prevention and Control. Weekly influenza surveillance overview [Internet]. Stockholm: ECDC; c2005-2016 [cited 2016 Mar 14]. Available from: http://ecdc.europa.eu/en/publications/surveillance_reports/influenza/Pages/weekly_influenza_surveillance_overview.aspx? $\mathrm{p}=15$.

9. Review of the 2010-2011 winter influenza season, northern hemisphere. Wkly Epidemiol Rec. 2011 May 27;86(22):222-7.

10. Review of the 2011-2012 winter influenza season, northern hemisphere. Wkly Epidemiol Rec. 2012 Jun 15;87(24):233-40.

11. Review of the 2012-2013 winter influenza season, northern hemisphere. Wkly Epidemiol Rec. 2013 May 31;88(22):225-32.

12. Review of the 2013-2014 winter influenza season, northern hemisphere. Wkly Epidemiol Rec. 2014 Jun 6;89(23):245-56.

13. Hay AJ, Gregory V, Douglas AR, Lin YP. The evolution of human influenza viruses. Philos Trans R Soc Lond B Biol Sci. 2001 Dec 29;356(1416):1861-70.
14. Barr IG, McCauley J, Cox N, Daniels R, Engelhardt OG, Fukuda K, et al.; Writing Committee of the World Health Organization Consultation on Northern Hemisphere Influenza Vaccine Composition for 2009-2010. Epidemiological, antigenic and genetic characteristics of seasonal influenza $\mathrm{A}(\mathrm{H} 1 \mathrm{~N} 1), \mathrm{A}(\mathrm{H} 3 \mathrm{~N} 2)$ and $\mathrm{B}$ influenza viruses: basis for the WHO recommendation on the composition of influenza vaccines for use in the 2009-2010 northern hemisphere season. Vaccine. 2010 Feb 3;28(5):115667.

15. Bonmarin I, Belchior E, Le Strat Y, Levy-Bruhl D. First estimates of influenza vaccine effectiveness among severe influenza cases, France, 2011/12. Euro Surveill. 2012 May 3;17(18). pii: 20163.

16. Lee BE, Mukhi SN, Drews SJ. Association between patient age and influenza A subtype during influenza outbreaks. Infect Control Hosp Epidemiol. 2010 May;31(5):535-7.

17. Beauté J, Broberg E, Plata F, Bonmarin I, O Donnell J, Delgado C, et al. Overrepresentation of influenza A(H1N1)pdm09 virus among severe influenza cases in the 2011/12 season in four European countries. Euro Surveill. 2012 Mar 1;17(9). pii: 20105.

18. Pariani E, Amendola A, Ebranati E, Ranghiero A, Lai A, Anselmi G, et al. Genetic drift influenza $\mathrm{A}(\mathrm{H} 3 \mathrm{~N} 2)$ virus hemagglutinin (HA) variants originated during the last pandemic turn out to be predominant in the 2011-2012 season in Northern Italy. Infect Genet Evol. 2013 Jan;13:25260.

19. Punpanich W, Chotpitayasunondh T. A review on the clinical spectrum and natural history of human influenza. Int J Infect Dis. 2012 Oct;16(10):e714-23.

20. Wilking AN, Elliott E, Garcia MN, Murray KO, Munoz FM. Central nervous system manifestations in pediatric patients with influenza A H1N1 infection during the 2009 pandemic. Pediatr Neurol. 2014 Sep;51(3):3706.

21. Goenka A, Michael BD, Ledger E, Hart IJ, Absoud M, Chow G, et al. Neurological manifestations of influenza infection in children and adults: results of a National British Surveillance Study. Clin Infect Dis. 2014 Mar;58(6):775-84.

22. Gutiérrez-Pizarraya A, Pérez-Romero P, Alvarez R, Aydillo TA, OsorioGómez G, Milara-Ibáñez C, et al. Unexpected severity of cases of influenza $\mathrm{B}$ infection in patients that required hospitalization during the first postpandemic wave. J Infect. 2012 Nov;65(5):423-30.

23. Mytton OT, Rutter PD, Donaldson LJ. Influenza A(H1N1)pdm09 in England, 2009 to 2011: a greater burden of severe illness in the year after the pandemic than in the pandemic year. Euro Surveill. 2012 Apr 5;17(14). pii: 20139.

24. Lehners N, Geis S, Eisenbach C, Neben K, Schnitzler P. Changes in severity of influenza $\mathrm{A}(\mathrm{H} 1 \mathrm{~N} 1)$ pdm09 infection from pandemic to first postpandemic season, Germany. Emerg Infect Dis. 2013 May; 19(5):74855.

25. GISAD [Internet]. The GISAD Initiative; 2016 [cited 2016 March 14]. Available from: http://platform.gisaid.org/.

Received February 5, 2015 Accepted in revised form March 14, 2016 